\title{
La información climático-meteorológica proporcionada al turista. Explorando el caso español ${ }^{1}$
}

\author{
María Belén GÓMEZ MARTíN \\ Departamento de Geografía Física y Análisis Geográfico Regional \\ Universidad de Barcelona \\ bgomez@ub.es \\ Xosé A. ARMesto LóPEZ \\ Departamento de Geografía Física y Análisis Geográfico Regional \\ Universidad de Barcelona \\ Emilio MARTÍNEZ IBARRA \\ Departamento de Análisis Geográfico Regional y Geografía Física \\ Universidad de Granada
}

Recibido: 21 de mayo del 2014

Enviado a evaluar: 28 de mayo del 2014

Aceptado: 17 de julio del 2014

\section{RESUMEN}

La relevancia que el tiempo y el clima desempeñan en la planificación de los destinos y en la experiencia de viaje, nos advierte de la importancia que adquiere la información climático-meteorológica para el normal desarrollo de la actividad. Proporcionar información adaptada a los distintos usuarios y necesidades, con diferente resolución temporal y espacial, puede ser un elemento clave para el éxito y la sostenibilidad de los destinos turísticos. Este artículo explora, para el caso español, las características y deficiencias existentes en la información climático-meteorológica proporcionada a los turistas. Para ello se analizan los portales de los principales servicios públicos y privados de información climáticometeorológica y los portales promocionales de los destinos españoles a nivel estatal y autonómico. Los resultados indican que la información climático-meteorológica proporcionada a los turistas aunque aprueba los requisitos de disponibilidad, fiabilidad y credibilidad marcados por la OMM, no supera al completo los relativos al criterio de utilidad, por las carencias en la adaptación a las necesidades del turista consumidor.

Palabras clave: información climático-meteorológica, turistas, destinos, Tecnologías de la Información y de la Comunicación (TICs), España.

${ }^{1}$ Este aportación ha sido realizada en el marco del proyecto de investigación del Plan Nacional de $\mathrm{I}+\mathrm{D}+\mathrm{i}$ del Ministerio de Ciencia e Innovación del Gobierno de España, con referencia CSO2011-23404. 


\title{
Climate and meteorological information to tourists. Exploring the Spanish case
}

\begin{abstract}
The importance of the weather and climate for the planning of tourist destinations and the overall travel experience highlights the significance of climate and meteorological information for the trouble-free development of these activities. Providing information at different temporal and spatial resolutions that satisfies different users and their varying needs can be essential to ensure the success and sustainability of tourist destinations. This article examines the characteristics and shortcomings of the climate and meteorological information provided to tourists in the Spanish case. In doing so, we analyse the portals of the main public and private climate and meteorological information services and the portals promoting Spain's tourist destinations at both the national and regional levels. The results indicate that the climate and meteorological information provided to tourists, while fulfilling the requirements of availability, reliability and credibility established by the WMO, fail to fully satisfy utility requirements as it does not always adapt to the needs of the tourist consumer.
\end{abstract}

Key words: climate and meteorological information, tourists, tourist destinations, Information and Communication Technologies (ICTs), Spain .

\section{L'information climatico-météorologique aur touriste. Exploration du cas espagnol}

\section{RÉSUMÉ}

L'importance du temps et du climat dans la planification des destinations et de l'expérience du voyage nous avise de l'importance qu'acquiert l'information climatico-météorologique pour le développement normal de cette activité. Fournir l'information adaptée aux divers usagers et nécessités, avec différentes résolutions temporelles et spatiales, peut être un élément clé pour le succès et la durabilité des destinations touristiques. Cet article explore, pour le cas espagnol, les caractéristiques ainsi que les déficiences existant dans l'information climatico-météorologique fournie aux touristes. Pour ce faire, il analyse les portails des principaux services publics et privés d'information climatico-météorologique ainsi que les portails promotionnels des destinations espagnoles aux niveaux du pays et des communautés autonomes. Les résultats indiquent que l'information climatico-météorologique fournie aux touristes, bien qu'elle réponde aux conditions de disponibilité, de fiabilité et de crédibilité marqués par l'Organisation météorologique mondiale (OMM), ne dépassent pas l'ensemble de celles relatives au critère d'utilité, à cause des carences en termes d'adaptation aux besoins du touriste consommateur.

Mots clés : information climatico-météorologique, touristes, destinations, Technologies de l'Information et de la Communication (TIC), Espagne.

\section{INTRODUCCIÓN}

La importancia del turismo en la economía global y los innegables vínculos que la actividad manifiesta con los elementos atmosféricos aconsejan el estudio del binomio turismo - clima en sus múltiples facetas. Si bien es cierto que en los últimos años se ha producido un crecimiento espectacular en el número de contribuciones científicas en este campo (Hall y Higham, 2005; Martínez Ibarra y Gómez Martín, 2012; Scott, Wall y McBoyle, 2005, Scott y Lemieux, 2010) restan todavía muchos aspectos por 
investigar, entre ellos el relativo a la información climático-meteorológica proporcionada al turista.

Las interrelaciones que se establecen entre el turismo y el clima son variadas y complejas (Becken et al., 2013; Gómez Martín, 2005), siendo los condicionantes atmosféricos aspectos clave en la planificación de los destinos turísticos y en la experiencia de viaje (Becken, 2010; Hamilton et al., 2005).

Desde el punto de vista de la planificación, cabe señalar que el tiempo meteorológico, la variabilidad climática intra e interanual, los fenómenos meteorológicos extremos o el cambio climático son elementos básicos a tener en cuenta en las regiones-destino de los flujos turísticos por la influencia que ejercen en la competitividad y en la sostenibilidad, tanto a corto como a largo plazo. En este sentido, el tiempo meteorológico condiciona, según la mayor o menor sensibilidad de la modalidad turística practicada hacia los elementos atmosféricos, la programación y el normal desarrollo de las actividades turísticas (Gómez Martín et al., 2012; Mckercher et al., 2014; Moreno et al., 2008; Shih et al., 2009). Del mismo modo, en los destinos turísticos, la variabilidad climática intra e interanual ejerce un notable influjo en la estacionalidad turística, el abastecimiento de agua, la producción artificial de nieve, el gasto energético o las necesidades de riego o ajardinamiento, por citar tan solo algunos ejemplos (Martínez Ibarra et al., 2012; Ridderstaat et al., 2014; Rico Amoros et al., 2009). Los fenómenos meteorológicos extremos (olas de calor, inundaciones, sequías, etc.) comprometen, en diferente medida, la seguridad en los destinos por lo que deben ser considerados en los sistemas de alerta temprana, la comunicación de riesgos a los agentes turísticos y a los turistas y, por supuesto, en la ordenación de los destinos (Bigano et al., 2005; Gómez Martín et al., 2013). Así pues, el tiempo y el clima son elementos clave para el turismo por lo que cualquier cambio en las condiciones atmosféricas actuales puede comportar impactos de diverso signo para el sector, en algunos casos muy significativos (Becken et al., 2007; Hall et al., 2005; Scott et al., 2008).

Por otro lado, el tiempo meteorológico y el clima tienen un peso destacado en la toma de decisiones del turista-consumidor y en la experiencia de viaje. Los aspectos atmosféricos, tanto del lugar de origen como del lugar de destino, son importantes elementos motivadores para el turista y son tenidos muy en cuenta en la planificación del viaje (Eugenio Martín et al., 2010; Gössling et al., 2012, Hu et al., 1993). Además, el tiempo y el clima condicionan la experiencia turística por lo que influyen en la satisfacción del visitante (Bigano et al., 2006). Todo ello hace que el clima se convierta en un componente destacado de la imagen del destino por el enorme poder de atracción que ejerce sobre el turista-consumidor (Andreu et al., 2000; Gómez Martín, 1999; Pike, 2002).

\subsection{INFORMACIÓN CLIMÁTICO-METEOROLÓGICA Y TURISMO}

La relevancia que el tiempo y el clima desempeñan en la planificación de los destinos y en la experiencia de viaje, nos advierte de la importancia que adquiere la información climático-meteorológica para el normal desarrollo de la actividad (Altalo 
et al., 2002; De Freitas, 2003). Proporcionar información adaptada a los distintos usuarios y necesidades, con diferente resolución temporal y espacial, puede ser un elemento clave para el éxito y la sostenibilidad de los destinos turísticos (Figura 1).

Figura 1. Información climático-meteorológica y turismo.

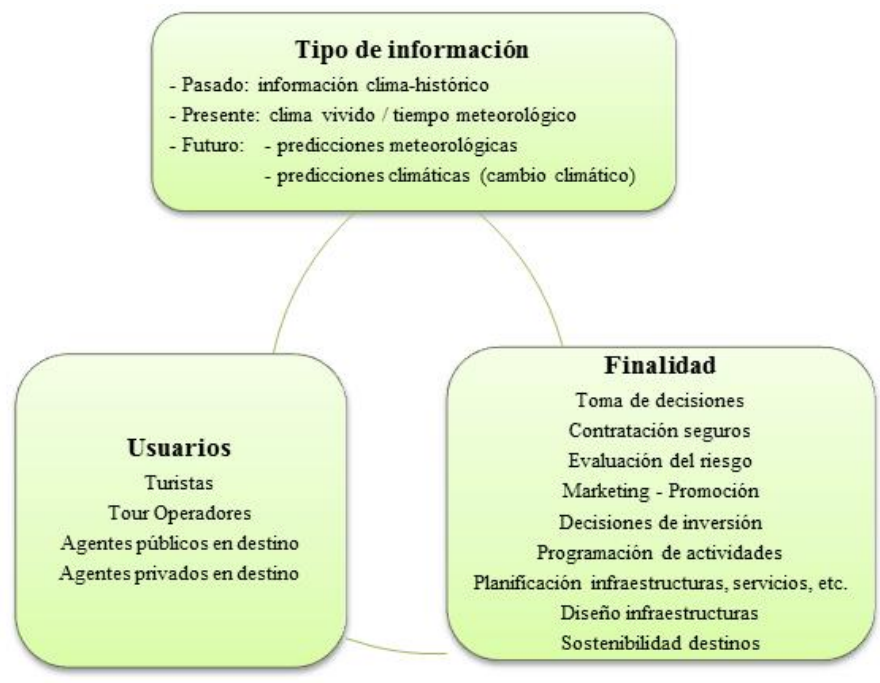

Fuente: Elaboración propia.

De los posibles usuarios de información climático-meteorológica que podrían ser abordados aquí, este artículo se centra en el turista. Tal y como señalan Scott y Lemieux (2010) la información climático-meteorológica que se le proporciona al consumidor merece una especial atención por el papel fundamental que juega en las diferentes fases de la experiencia turística (Figura 2).

Así, durante el período pre-vacacional tiene lugar la planificación del viaje por parte del turista. El clima y el tiempo meteorológico suelen ser importantes elementos motivadores por lo que las condiciones atmosféricas en el lugar de origen actúan como marco en el que se inscribe la toma de decisiones. En esta fase, el potencial turista trata de elegir el destino y el momento en el que viajar y, para ello, tiene en cuenta fundamentalmente la información climática de los lugares barajados. Esta información, adquirida a través de diferentes vías -conocimientos propios, guías de viaje, folletos informativos, portales promocionales, web 2.0, etc.-, le permiten tomar una decisión durante los meses previos y realizar la compra. A medida que se aproxima la fecha de viaje ( 2 o 3 semanas), el turista empieza a preocuparse por el tiempo meteorológico del lugar de destino, por lo que se convierte en un seguidor incansable de las predicciones meteorológicas que ofrecen los medios de comunicación y los organismos públicos y/o privados que ofrecen este tipo de servicio. La información meteorológica y las predicciones le sirven para realizar la 
planificación de actividades, la elección de rutas o itinerarios, la preparación del equipaje, etc. Esta información también es la utilizada por los turistas que reservan a última hora, un segmento del mercado que ha experimentado un espectacular crecimiento en la última década.

Durante el período vacacional tiene lugar el viaje propiamente dicho. El turista en el destino se preocupa por las condiciones atmosféricas vividas, es decir por el tiempo meteorológico. Estas y las predicciones para los días inmediatos le ayudan a programar sus actividades, determinan su comportamiento, su nivel de gasto, su seguridad, disfrute y confort. La experiencia vivida proporciona al turista una primera impresión de satisfacción.

Por último, finalizado el viaje y ya en el lugar de origen, el turista -a partir de sus recuerdos- realiza un balance entre la experiencia atmosférica vivida y la experiencia esperada. Este balance, realizado de nuevo en el marco ambiental del lugar de origen, determina -en parte- su nivel de satisfacción y condiciona su fidelidad y su papel promotor del destino.

Figura 2. Influencia de la información climático-meteorológica en las decisiones del turista.

\begin{tabular}{|c|c|c|c|c|}
\hline & \multicolumn{2}{|c|}{$\begin{array}{c}\text { Período } \\
\text { pre-vacacional }\end{array}$} & Período vacacional & $\begin{array}{c}\text { Período } \\
\text { post-vacacional }\end{array}$ \\
\hline & \multicolumn{2}{|c|}{ Planificación del viaje } & Viaje & Valoración del viaje \\
\hline $\begin{array}{c}\text { Decisiones del } \\
\text { turista }\end{array}$ & $\begin{array}{l}\text { Motivación viaje } \\
\text { Selección } \\
\text { destino } \\
\text { Época viaje } \\
\text { Contratación } \\
\text { seguros }\end{array}$ & $\begin{array}{c}\text { Selección } \\
\text { destino en } \\
\text { reservas } \\
\text { "última hora" } \\
\text { Planificación } \\
\text { actividades } \\
\text { Itinerario/Rutas }\end{array}$ & $\begin{array}{c}\text { Programación de actividades } \\
\text { Disfrute } \\
\text { Seguridad } \\
\text { Confort } \\
\text { Gasto turístico } \\
\text { Satisfacción }\end{array}$ & $\begin{array}{l}\text { Recuerdos } \\
\text { Satisfacción } \\
\text { Fidelización } \\
\text { Recomendación }\end{array}$ \\
\hline \multirow[t]{2}{*}{ Tiempo/Clima } & \multicolumn{2}{|c|}{ 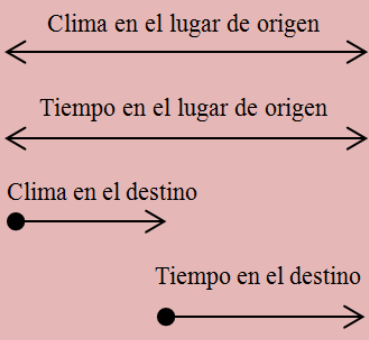 } & $\leftarrow$ Tiempo en el destino & 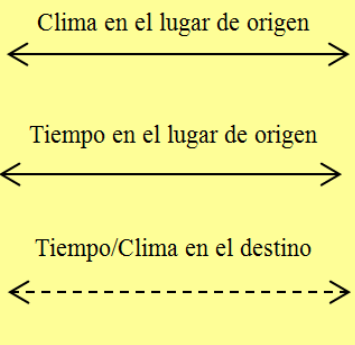 \\
\hline & Meses & Día & Semana & Meses \\
\hline
\end{tabular}

Fuente: Adaptado y modificado a partir de la propuesta realizada por Scott y Lemieux (2010).

Así pues, el turista-consumidor requiere información climático-meteorológica en las diferentes fases de la experiencia turística. Para que su vivencia se lleve a cabo de forma satisfactoria, no sólo necesita del cumplimiento de sus expectativas climáticometeorológicas, sino que también exige del cumplimiento de unos requisitos informativos. De este modo, el turista reclama a la información climático- 
meteorológica cuatro características fundamentales (en terminología de la Organización Meteorológica Mundial -OMM-): a) disponibilidad: el turista debe recibir la información que necesita y cuando la necesita; b) fiabilidad: el turista debe recibir la información puntualmente y sin falta; c) credibilidad: el turista utilizará la información que se le proporciona si considera que es correcta $\mathrm{y}$, en ello, la fuente desempeña un papel fundamental; y d) utilidad: el turista necesita la información adaptada a su nivel y a sus necesidades. En el cumplimiento de estos requisitos juegan un papel fundamental las fuentes, los proveedores de información y las Tecnologías de la Información y de la Comunicación (TICs).

\subsection{FUENTES, PROVEEDORES DE INFORMACIÓN CLIMÁTICO- METEOROLÓGICA Y TECNOLOGÍAS DE LA INFORMACIÓN Y DE LA COMUNICACIÓN}

En las últimas dos décadas, las fuentes, los proveedores de información climáticometeorológica y las formas de llegar a los usuarios finales (en nuestro caso, el turistaconsumidor) se han diversificado notablemente.

Prácticamente, todos los países cuentan con una o varias agencias de carácter público que tienen la responsabilidad de suministrar servicios y/o productos de información meteorológica y climática a diversos usuarios finales. Así, por ejemplo, España cuenta en la actualidad con una agencia a escala estatal y tres a escala autonómica. A estos organismos públicos, se suman aquellos de carácter privado que han proliferado debido al alto valor añadido y comercial que se puede derivar de este tipo de servicios. Como fuentes de información primaria también se deben señalar diversas entidades dedicadas a la investigación o al turismo (estaciones de esquí, estaciones náuticas) que generan datos de utilidad adecuados a sus intereses.

Esta información primaria es difundida por diversos proveedores. Muchas veces, las propias agencias y organismos que generan la información se encargan de difundirla a los usuarios finales. Otras, sin embargo, existen intermediarios que realizan esa labor de difusión. En este sentido, cabe señalar el papel desempeñado por algunas de las herramientas promocionales e informativas de los propios destinos (folletos turísticos, portales de promoción y comercialización del destino, paneles informativos en destino, etc.) o determinadas asociaciones turísticas.

En cuanto a las formas de llegar a los usuarios-turistas cabe señalar que a los tradicionales medios de comunicación personales se suman hoy los medios de comunicación de masas, dentro de los cuales Internet asume un protagonismo indiscutible. La OMM señala que, sin subestimar la importancia de los beneficios relacionados con los progresos científicos y la capacidad de cálculo informático a lo largo de la última década, el avance que ha sido más útil para la prestación de servicios meteorológicos y climáticos desde el punto de vista de los usuarios ha sido el protocolo WWW (OMM, 2008; 2012). En este sentido, se puede afirmar que éste ha facilitado el acceso al conocimiento y a la información en cualquier momento y lugar, posibilitando la integración del usuario en procesos de toma de decisiones diseñados para él. 
Así pues, las TICs han permitido el desarrollo de nuevas formas de recopilar, usar y transmitir la información en tiempo real, y ello es importante para el prestador de servicios y también para el usuario-receptor, en nuestro caso el turista. En este sentido, el auge de la comunicación en movilidad -propiciada por la aparición y desarrollo de la telefonía móvil- y las posibilidades de interactividad que ofrece la Red -redes sociales, web 2.0- (Aldeanueva et al., 2012; Antón Clavé et al., 2004) ayudan a explicar que Internet se haya convertido en la segunda fuente de información para la toma de decisiones de los viajeros españoles, tras la recomendación de amigos y familiares (Tabla 1).

Tabla 1. ¿Cuáles de las siguientes fuentes de información le llevaron a elegir destino? (Respuesta múltiple)

\begin{tabular}{|l|c|}
\hline \multicolumn{1}{|c|}{ Fuente de información } & \% \\
\hline Recomendación amigos/familiares & 53 \\
\hline Recomendación Ferias de viajes & 6 \\
\hline Recomendación Agencia de Viajes física & 4 \\
\hline Recomendación Oficina de Turismo & 2 \\
\hline Buscador de Internet & 28 \\
\hline Agencia de Viajes on line & 6 \\
\hline Web de destinos turísticos & 4 \\
\hline Página de proveedores de viaje & 3 \\
\hline RRSS, foros y blogs & 1 \\
\hline TV anuncios & 3 \\
\hline MM impresos (folletos) & 3 \\
\hline Radio & 1 \\
\hline
\end{tabular}

Fuente: Lookinside.travel, 2013.

Pero la influencia de Internet, la tecnología móvil y la web 2.0 van mucho más allá de la búsqueda de información para la toma de decisiones en el período prevacacional (Figura 3), adquiriendo también un protagonismo indiscutible en el período vacacional y post-vacacional (Chung et al., 2008; Lim et al., 2010). Así, por ejemplo, el número y la variedad de actividades relacionadas con los viajes que se realizan a través del móvil en el lugar de vacaciones aumenta año tras año ${ }^{2}$, siendo la búsqueda de mapas/direcciones, información del destino (como por ejemplo, información meteorológica ${ }^{3}$ ), actualización de redes sociales, búsqueda de productos del viaje y la descarga de aplicaciones del destino las actividades más destacadas

${ }^{2}$ La tasa de viajeros españoles con Smartphones es del 61\% (Lookinside.travel, 2013).

${ }^{3}$ En España, las aplicaciones -para distintas plataformas de teléfonos móviles inteligentesrelacionadas con servicios de información meteorológica son de las más descargadas por los usuarios $(29 \%)$, junto con las relativas a redes sociales $(44 \%)$, localizadores $(33 \%)$, noticias (30\%) y música (30\%) (Nielsen, 2011). 
realizadas por los viajeros españoles, según el último informe del Lookinside.travel (2013).

En la fase post, los usuarios también suelen hacer uso de la Red, esta vez para proporcionar información -por ejemplo sobre el tiempo/clima- a otros turistas, compartiendo experiencias, emitiendo opiniones y recomendaciones, subiendo fotografías, etc., que pueden resultar de utilidad a otros visitantes (Figura 3). Los comentarios emitidos por los turistas influyen en la toma de decisiones de otros turistas debido fundamentalmente a su carácter experiencial y motivador. Tal y como señalan Prahalad y Ramaswamy "los usuarios ya no están dispuestos a aceptar experiencias fabricadas por las empresas. Cada vez más, ellos quieren cambiar o modelar estas experiencias por ellos mismos, ya sea individualmente o a través de la experiencia de otros consumidores" (cit. en Huertas, 2008: 2).

Figura 3. Principales medios de adquisición/transmisión de información climáticometeorológica.

\begin{tabular}{|c|c|c|c|c|}
\hline & \multicolumn{2}{|c|}{$\begin{array}{c}\text { Periodo } \\
\text { pre-vacacional }\end{array}$} & Periodo vacacional & $\begin{array}{c}\text { Periodo } \\
\text { post-vacacional }\end{array}$ \\
\hline & \multicolumn{2}{|c|}{ Planificación del viaje } & Viaje & Valoración del viaje \\
\hline \multirow{2}{*}{ Turista } & \multicolumn{2}{|c|}{ Demanda información } & Demanda información & Proporciona información \\
\hline & Climática & Meteorológica & Meteorológica & Climática/Meteorológica \\
\hline \multirow[t]{2}{*}{$\begin{array}{l}\text { Principales Medios de } \\
\text { adquisición/transmisión } \\
\text { de la información } \\
\text { climático-meteorológica }\end{array}$} & $\begin{array}{c}\text { Guias de viajes } \\
\text { Folletos } \\
\text { Conocimientos } \\
\text { previos } \\
\text { TT.OO } \\
\text { Agencias Viajes } \\
\text { Web } 2.0\end{array}$ & $\begin{array}{l}\text { Internet } \\
\text { Prensa } \\
\text { Tv } \\
\text { Radio }\end{array}$ & $\begin{array}{c}\text { Información in situ } \\
\text { Internet } \\
\text { Apps } \\
\text { Prensa } \\
\text { TV } \\
\text { Radio }\end{array}$ & $\begin{array}{c}\text { Web 2.0.: } \\
\text { Blogs } \\
\text { Redes sociales } \\
\text { Agregadores de noticias, } \\
\text { Imágenes (Flickr, Instagram,etc.) }\end{array}$ \\
\hline & Meses & Dia & Semana & Meses \\
\hline
\end{tabular}

Fuente: Elaboración propia.

La evolución de las tecnologías ha aumentado las posibilidades de acceso a la información por parte de los usuarios en cualquier momento y lugar. En el caso del turista, el mejor acceso a una información meteo-climática de calidad podría convertirse en un elemento favorecedor de "la interacción y la integración del usuario en el destino que contribuiría a incrementar la calidad de la experiencia turística", referente clave de los llamados Nuevos Destinos Turísticos Inteligentes, considerados medida prioritaria dentro del Plan Nacional e Integral de Turismo 2012-2015 del Gobierno de España (Ivars, 2012: 475). 


\section{OBJETIVO Y METODOLOGÍA}

El número de fuentes, proveedores y las ventajas que ofrece la navegación de gran alcance podrían hacernos pensar que la información climático-meteorológica existente es suficiente para los actuales usuarios-turistas. Sin embargo, nuestra hipótesis de partida sostiene que la información que se ofrece no tiene demasiado en cuenta las necesidades del turista. En este sentido, planteamos que si bien la información climático-meteorológica proporcionada en la Red aprueba los requisitos de disponibilidad, fiabilidad y credibilidad marcados por la OMM, no supera los relativos al criterio de utilidad. Teniendo en cuenta nuestra hipótesis de partida, este artículo explora, para el caso español, las características y deficiencias existentes en la información climático-meteorológica proporcionada a los turistas con objeto de detectar fortalezas y debilidades que permitan avanzar en la gestión de los destinos y la calidad de la experiencia turística. Para ello se analizan los contenidos proporcionados por tres tipos de proveedores: Servicios Nacionales y Autonómicos de información sobre clima y tiempo, Servicios privados de información climáticometeorológica y organismos encargados de la promoción turística de los destinos a nivel estatal y autonómico. El carácter de los proveedores analizados es muy diferente. Por un lado, los dos primeros como suministradores de servicios que son -públicos o privados- tratan de adaptarse en la medida de lo posible a los intereses de los diferentes usuarios potenciales. En cambio, los organismos encargados de la promoción tratan de responder a sus propios intereses de marketing. Quedan al margen de este análisis otros organismos específicos que también generan o difunden este tipo de información (asociaciones turísticas relacionadas con alguna actividad en concreto, patronatos de turismo, etc.).

Con objeto de examinar el cumplimiento del criterio de utilidad señalado por la OMM, el análisis de contenido aplicado en este trabajo evalúa en los portales analizados los siguientes aspectos de la información climática y meteorológica:

1. Interés de la información para el turista: número de parámetros atmosféricos y ambientales proporcionados.

2. Grado de sistematización de la información: agrupación de la información con criterios turísticos, por ejemplo, modalidades turísticas, ámbitos turísticos, etc.

3. Adaptación al nivel de usuario: utilización de un lenguaje inteligible, no demasiado técnico, con infografía asociativa.

4. Adaptación a las necesidades del usuario: información para distintas modalidades turísticas, integrada (formulación de tipos de tiempo), con indicativos de aptitud, con valores de probabilidad (y no valores medios), con diferente resolución temporal (horaria, predicciones para varios días, etc.) y que, además, incluya consejos, webcams, apps, etc. 


\section{LA INFORMACIÓN CLIMÁTICO-METEOROLÓGICA PARA EL TURISTA}

\subsection{PROVEEDORES PÚBLICOS DE INFORMACIÓN CLIMÁTICO- METEOROLÓGICA: SERVICIOS NACIONALES Y AUTONÓMICOS}

Cuatro son las agencias de meteorología que dependen de organismos oficiales de la Administración del Estado o las Comunidades Autónomas: la Agencia Estatal de Meteorología (AEMET), el Servicio Meteorológico de Cataluña / Servei Meteorològic de Catalunya (MeteoCat), la Agencia Vasca de Meteorología / Euskal Meteorologia Agentzia (Euskalmet) y MeteoGalicia. Todas ellas tienen como objetivo el desarrollo, la implantación y prestación de servicios meteorológicos y climáticos, así como el apoyo al ejercicio de diversas políticas públicas y actividades privadas que puedan contribuir a la seguridad, bienestar y desarrollo sostenible de la sociedad. Es por ello que todas han realizado un notable esfuerzo de adaptación a los diferentes usuarios potenciales de este tipo de información, entre ellos el usuario con necesidades turísticas. En este sentido, se observa que todos los portales, en mayor o menor medida, contienen información meteorológica de interés para el visitante turístico (se ofrece -como corresponde a este tipo de servicios- un elevado número de parámetros meteorológicos y ambientales), pero la misma no siempre se encuentra sistematizada (por ámbito turístico o por modalidad turística), ni adaptada al nivel del usuario (aunque se emplea con frecuencia una cuidada infografía, la explicación descriptiva-verbal sigue siendo muy técnica), ni a sus necesidades, al menos de forma gratuita. En cuanto a este último criterio, cabe señalar que si bien en todos los portales quedan cubiertas las necesidades de información para diferentes momentos horarios y días vista, no se incorporan valores de aptitud para la práctica de diferentes modalidades turísticas, ni la información aparece integrada en tipos de tiempo, ni se proporcionan valores probabilísticos. Rara vez se facilitan consejos -salvo contadas excepciones-, o Apps y webcams que ofrezcan imágenes en tiempo real. En cuanto a la información climática contenida en todos los portales visitados, cabe señalar que tampoco resulta de utilidad al usuario turista y suele ser de difícil acceso.

Así, para el caso de AEMET, cabe señalar que contempla los principales escenarios o ámbitos turísticos del país: el litoral, la montaña y el urbano. En cuanto al ámbito litoral, el portal ofrece información meteorológica para las principales playas, cubriendo las necesidades que pudieran demandar los turistas atraídos por la modalidad estrella. Dentro del ámbito litoral, la Web también ofrece información de detalle para aquellos turistas interesados en la práctica de deportes náuticos a través del Boletín marítimo y meteorológico para zonas costeras y alta mar y el sistema de información METEONAV que muestra vientos y diferentes tipos de oleaje de interés para la navegación marítima, costera u oceánica.

En cuanto al ámbito turístico de la montaña, el portal presenta información para diferentes áreas a dos niveles: a) predicción meteorológica (para nueve áreas de montaña) y b) predicción nivológica (se restringe al ámbito pirenaico, durante el período que va de diciembre a mayo). Para el ámbito urbano, existe un apartado que 
también puede interesar al turista en el momento de planificar su viaje a ciudades bien del propio país, bien del extranjero. Así, desde el menú lateral izquierdo se puede acceder a la sección Ciudades del mundo y a partir de ahí la página muestra el link con el Servicio de Información Meteorológica Mundial, donde se puede realizar la consulta correspondiente.

AEMET también incluye otras secciones de interés turístico. Así, por ejemplo, desde abril de 2014 se puede consultar la nueva sección Meteoruta que pretende ser un visor de mapas, a través del cual el usuario puede obtener las diferentes variables meteorológicas que pueden afectar a la circulación rodada por todo el país. Otra sección de interés turístico es la correspondiente al sistema de Avisos, donde el usuario puede visualizar las zonas de España amenazadas por algún evento atmosférico de magnitud extraordinaria. Para concluir, la página de AEMET cuenta con una App oficial gratuita. En ella se puede consultar la previsión meteorológica a escala municipal de toda España, la información de los avisos por fenómenos adversos día a día y hora a hora, y el radar de precipitación. También se pueden memorizar lugares favoritos y el propio teléfono detecta la ubicación del usuario ofreciéndole el tiempo de ese lugar.

La página catalana Meteocat presenta rasgos comunes con la página estatal, dado que contiene información que puede ser de interés para el turista tanto en el ámbito litoral como en el ámbito de la montaña, o en el caso de los avisos meteorológicos. De todos modos, el grado de sistematización es menor que el del portal estatal y mayor la complejidad en el tratamiento y comunicación de la información. Así, aunque desde la página inicial se puede acceder fácilmente a la información de los Avisos meteorológicos de situación de riesgo, resulta complejo para un usuario medio, entender las magnitudes por las que se genera ese aviso. De este modo, por ejemplo, a un turista poco o nada le dice "superar el percentil 98 de la temperatura máxima durante tres días", si no se le facilita una magnitud concreta que él pueda entender y sea impactante (p.e. $40^{\circ} \mathrm{C}$ ). Para poder acceder a esta información más inteligible, el usuario debe seguir navegando por la página.

El portal de Euskalmet es el que presenta una menor adecuación de los servicios al perfil del usuario turístico. La información se muestra en bruto, sin hacer referencia a ningún escenario turístico en concreto o actividad. Lógicamente, el usuario puede obtener información de interés pero para ello debe navegar constantemente por la web.

Por último, MeteoGalicia incluye en su arquitectura referencias turísticas directas. Así pues, en su apartado de predicción, pueden tener interés para distintas tipologías de turistas los referentes a Avisos, Marítima, Caminos, Ultravioletas y Playas. Merece la pena comentar por su singularidad los apartados de Caminos y Ultravioleta, dado que Avisos, Marítima y Playas guardan gran semejanza con los presentados por AEMET y Meteocat. De este modo, en el apartado Caminos, mediante un mapa que contiene los distintos caminos jacobeos oficiales se representan gráficamente los meteoros, la temperatura actual y la sensación térmica para diferentes tramos. Además, el peregrino puede consultar la predicción para el día actual y para el día siguiente para las franjas de mañana y tarde. En cuanto a la sección Ultravioleta, cabe señalar que además de poder consultar los datos de los últimos cuatro días en las 
estaciones representativas de cuatro áreas turísticamente destacables del país y las predicciones, se ofrece información sobre los valores de tiempo máximo de exposición al sol según la escala Fitzpatrick y del factor de protección recomendado. Sin duda, una información muy interesante y útil para el turista o la persona que realice actividades en el exterior. En el caso de Meteogalicia, también hay que reseñar la existencia de dos Apps gratuitas. La primera de ellas, denominada MeteoSIX, es una aplicación que ofrece información meteorológica y oceanográfica; la segunda, denominada MeteoRoute, permite obtener la información meteorológica y oceanográfica personalizada para rutas y trayectorias, tanto por tierra como por mar.

\subsection{PROVEEDORES PRIVADOS DE INFORMACIÓN CLIMÁTICO- METEOROLÓGICA}

Durante los últimos años, el fulgurante desarrollo de las TICs ha permitido que en el sector privado hayan proliferado los servicios meteorológicos, ofreciendo una amplia gama de productos y aplicaciones para los usuarios turísticos finales (Scott y Lemieux, 2010). Ciertamente, los proveedores de información climática y meteorológica del sector privado adaptan sus productos a la demanda (WMO, 2002). Así, las compañías privadas ofrecen una gran cantidad de datos meteorológicos obtenidos de previsiones meteorológicas, tanto de acceso público como privado, adaptadas a destinos turísticos específicos, actividades turísticas particulares o subsectores turísticos determinados.

Con todo, a pesar de que a nivel internacional existen compañías privadas que ofrecen información meteorológica a los consumidores adaptadas a determinadas prácticas turísticas (el ejemplo más sobresaliente lo encontramos en EEUU, concretamente en la web "The Weather Channel"), en España no existe un servicio privado semejante. Bien es cierto que, por un lado, hay empresas como Meteosim o Meteora Servicios Meteorológicos y del Clima S.L. que ofrecen servicios específicos de predicción meteorológica para el sector turístico, si bien los mismos únicamente se ofrecen a empresas; y por otro que, para los consumidores, y para determinadas prácticas turísticas, especialmente dependientes del tiempo meteorológico, concretamente el surf y el esquí, existen páginas que ofrecen predicciones meteorológicas específicas. Así, para el surf podemos citar los siguientes ejemplos: "partedeolas.com", "olasinfo", "surfmediterráneo", "todosurf.com", "surfcantabria" o "valenciasurf.com", entre otras. Mientras que para el caso del esquí cabe traer a colación los que a continuación se enumeran: "infonieve.es” y "canalesquí". Con todo, en ningún caso de los citados se ofrece información especialmente útil y práctica para el consumidor, traduciendo la aptitud meteorológica de una jornada para una determina práctica turística en una escala de 1 a 10 , como si hace "The Weather Channel" para diversas prácticas turísticas, o "Surf-Forecast.com" para el caso del surf.

En España, los principales portales meteorológicos son "eltiempo.es", "eltiempotv.com", "tiempo.com" y "TuTiempo.net". En atención al tema que aquí preocupa, cabe resaltar que las 3 primeros ofrecen predicciones para 14 días 
(incluyendo el día en el que se realiza la consulta), mientras que "TuTiempo.net" lo hace para 15 días. Además, "eltiempotv.com" y "TuTiempo.net" son las únicas que ofrecen predicciones detalladas a medio-largo plazo (14 días) (Tabla 2).

Tabla 2. Información climática y meteorológica proporcionada por proveedores privados.

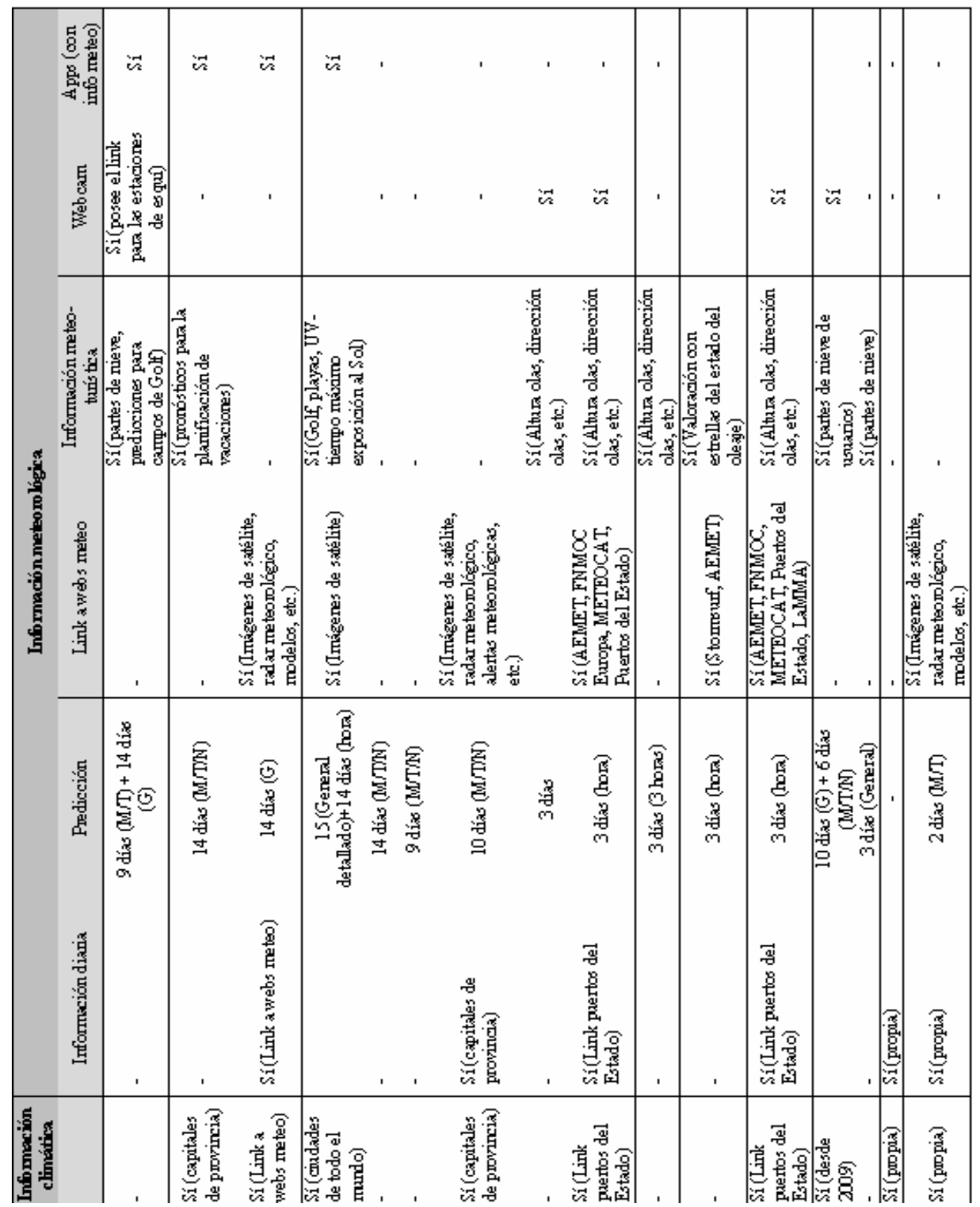

Fuente: Elaboración propia (consultas realizadas durante los meses de febrero, marzo y abril de 2014). 
Asimismo, cabe resaltar que las cuatro ofrecen Apps con información meteorológica. También conviene tener presente que "eltiempo.es", "eltiempotv.com" y "TuTiempo.net" ofrecen información meteorológica enfocada a la actividad turística, pero en ningún caso la misma se traduce a un índice climático-turístico diario de aptitud.

Además, cabe resaltar que en España existen dos redes de estaciones meteorológicas automáticas gestionadas por aficionados a la meteorología y de acceso gratuito. A nivel estatal aparece Meteoclimatic, y a nivel autonómico contamos con AVAMET (Asociación Valenciana de Aficionados a la Meteorología). Meteoclimatic ofrece datos de diversas variables meteorológicas (temperatura, precipitación, dirección y velocidad del viento, radiación solar y presión atmosférica), para un número elevado de localidades españolas. Por su parte, AVAMET ofrece datos meteorológicos en tiempo real de sus socios y de la propia asociación para el conjunto de la Comunidad Valenciana (temperatura real, temperatura máxima, temperatura mínima, humedad relativa, dirección y velocidad del viento y precipitación). También, permite el acceso libre a datos del tiempo pasado a escala diaria por municipios, agrupados, a su vez, por comarcas. En predicción meteorológica ofrece una prognosis a 24 horas vista para la Comunidad Valenciana, en forma de mapa significativo, acompaña de un texto sobre el cuadro sinóptico determinante y sus consecuencias meteorológicas.

\subsection{WEBS DE PROMOCIÓN TURÍSTICA DEL ESTADO Y COMUNIDADES AUTÓNOMAS}

Los organismos encargados de la promoción turística de los destinos suelen ser importantes proveedores de información climático-meteorológica. Suelen ser una de las principales fuentes a las que acuden los turistas en el momento de la toma de decisiones que se realiza durante la fase pre-vacacional (en especial el turista extranjero). Quizás por ello, la información climática ofrecida a través de los mismos no tiene demasiado en cuenta las necesidades del turista y se basa, fundamentalmente, en los intereses de marketing de estos proveedores de información. Estos portales también suelen ser consultados en destino, por la información meteo que contienen para el desarrollo de la experiencia durante la fase vacacional.

Las Webs de promoción turística del Estado así como las de las Comunidades Autónomas (CCAA) y Ciudades Autónomas (CA), analizadas según las categorías apuntadas en el apartado metodológico, evidencian que salvo contadas excepciones casi todas poseen algún tipo de información climática y/o meteorológica de sus destinos (Tabla 3). En relación a la información climática cabe señalar que tiene un menor peso que la información meteorológica. Concretamente, 9 de las 17 CCAA y una de las dos CA consultadas no poseen en sus portales ningún tipo de información sistematizada referente al clima, si bien las referencias al mismo se encuentran presentes de manera indirecta en las fotografías, los logotipos o en el cuerpo de texto informativo. Frente a ello, tan solo 2 de las 17 CCAA y una de las dos CA consultadas no poseen en sus portales ningún tipo de información meteorológica. 
Tabla 3. Información climática y meteorológica proporcionada por los portales de promoción turística de las Comunidades Autónomas y del Estado.

\begin{tabular}{|c|c|c|c|c|c|c|c|c|c|c|c|c|c|c|c|c|c|c|c|c|}
\hline \multirow{7}{*}{ है } & 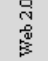 & is & $i$ & is & is & is & is & is & is is & is & is & is & is & is & is & $i s$ & is & is & is & in \\
\hline & 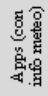 & & ' & & & $\overrightarrow{i n}$ & is & . & ' & . & is & ' & 溚 & is & & $\ddot{n}$ & $\ddot{\text { in }}$ & . & ' & ' \\
\hline & 丞 & & . & $\cdot$ & $\cdot$ & is & & $\therefore$ & $\begin{array}{lll}1 & 1\end{array}$ & 1 & is & 1 & ' & . & $\cdot$ & . & & . & ' & ' \\
\hline & 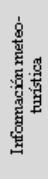 & & $\cdot$ & & ' & . & is & ' & $\begin{array}{lll} & 1 \\
\end{array}$ & ' & is & & 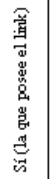 & 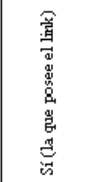 & & 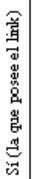 & 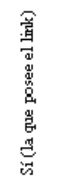 & . & ' & ' \\
\hline & 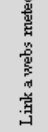 & & 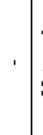 & 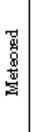 & & . 舅 & . & 1 & ' & ' & ' & ' & 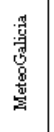 & 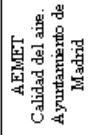 & & 槒 & $\begin{array}{l}\text { 蓄意 } \\
\text { 意湿 }\end{array}$ & . & ' & 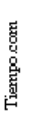 \\
\hline & 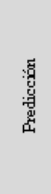 & 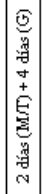 & 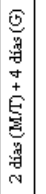 & & 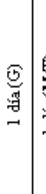 & 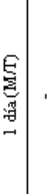 & 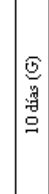 & & & 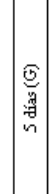 & 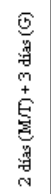 & 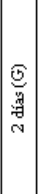 & 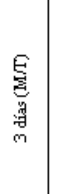 & ' & 㺃 & & ' & \begin{tabular}{|c|} 
\\
量 \\
m
\end{tabular} & & 总 \\
\hline & 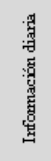 & 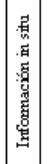 & 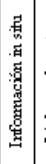 & 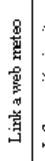 & 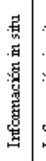 & 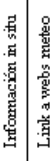 & 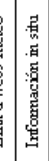 & 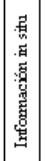 & & 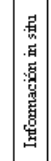 & 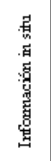 & 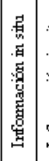 & 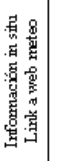 & 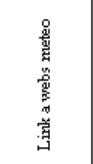 & 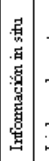 & 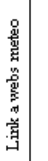 & 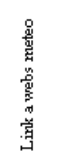 & 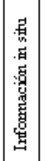 & & 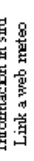 \\
\hline 夏 & & is & is & $\therefore$ & is & . & is & is & ' & is & ' & & . & is & $\therefore$ & $\therefore$ & & .1 & $\therefore$ & ' \\
\hline & & 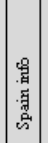 & 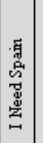 & 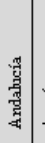 & 喜. & 解 & 勿 & 象 & 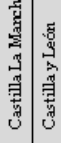 & 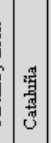 & 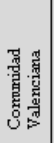 & 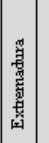 & ] & 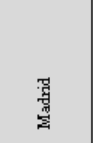 & 5 & 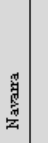 & 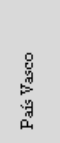 & $\mid \begin{array}{l}\frac{\pi}{3} \\
\frac{3}{3} \\
3\end{array}$ & 0 & \\
\hline
\end{tabular}

Fuente: Elaboración propia (consultas realizadas durante los meses de febrero, marzo y abril de 2014). 
Las webs que contienen información climática, realizan un enfoque descriptivoverbal de la información y tan sólo 4 de las 9 se atreven con tablas y valores numéricos de detalle mensual. Los parámetros referidos suelen ser las temperaturas (media de las máximas y media de las mínimas), las precipitaciones y la insolación, si bien algunas muestran datos sobre la temperatura del mar, velocidad del viento, radiación ultravioleta, humedad relativa y/o presión atmosférica. Es curioso observar que en las webs promocionales que utilizan un enfoque descriptivo-verbal existe un elevado grado de "generalización", “imprecisión" y/o "incorrección" de la información mostrada. Sirva como ejemplo las confusiones que existen en algunas de ellas entre temperatura media mensual y temperatura media de las máximas o lo que sucede con la insolación en la página de Spain.info: las "3000 horas de sol al año en España”, ocultan un gradiente nada despreciable comprendido entre las 1600 horas de la España Cantábrica, las referidas 3000 horas del sureste peninsular o las 3400 horas del archipiélago Canario.

Si bien es cierto que la información y los parámetros climáticos proporcionados en las diferentes webs consultadas pueden resultar de interés para el turista, no está del todo claro que puedan ser de utilidad. Es decir, son pocos los portales que ofrecen información climática adaptada a las necesidades del viajero. $\mathrm{Y}$ en este sentido, por ejemplo, no existe ningún portal que informe sobre calendarios de probabilidades de tipos de tiempo o calendarios de aptitud climática para las diferentes modalidades turísticas. Sólo el portal turístico de Canarias, en su sección elmejorclimadelmundo.com, ofrece una información adaptada al usuario, con herramientas comparadoras, consejos para aprovechar los beneficios del clima, opinión de expertos, etc.

En cuanto a la información meteorológica, 11 de las 15 webs consultadas poseen información in situ y 5 poseen links a webs proveedoras de este tipo de servicios. Tan sólo el portal gallego y el de la ciudad de Melilla presentan ambas posibilidades. Por lo general, la información proporcionada in situ es muy escueta y se limita al dato de temperatura máxima, temperatura mínima, probabilidad de precipitación, estado del cielo y predicción de esos parámetros básicos para $1 / 3$ días (Tabla 3). Son pocos los portales que ofrecen valores sobre índice UV, sensación térmica, velocidad y dirección del viento, temperatura del agua, etc. De las 15 webs analizadas, tan sólo 7 poseen información adaptada al turista ( 5 de estas 7 son links a webs proveedoras de este tipo de servicios). Los formatos son variados: desde información adaptada al tipo de actividad (por ejemplo, deportes náuticos, sol y playa, montaña, etc.), información adaptada al ámbito en el que se desarrolla la actividad (por ejemplo, turismo urbano) o información adaptada pero de carácter generalista. En cuanto a la presencia de webcams y Apps con información atmosférica adaptada al turista, señalar que poco a poco se van introduciendo en los portales promocionales, aunque no de modo generalizado ni gratuito (Tabla 3). En cambio, en los portales promocionales, sí que obtienen un mayor protagonismo las diferentes herramientas de la Web 2.0 (Tabla 3). 


\section{CONCLUSIONES}

Existe una diferencia notable entre la información atmosférica que proporcionan los organismos públicos y privados dedicados a suministrar servicios de información meteo-climática y la que facilitan los organismos encargados de la promoción de los destinos turísticos españoles. En los segundos existe una clara limitación ya que la información proporcionada no siempre contribuye a una mejor toma de decisiones, tácticas y estratégicas, en todas aquellas actividades que lleva a cabo el visitante y en las que el tiempo o el clima resultan relevantes. La información atmosférica ofrecida no tiene demasiado en cuenta los requerimientos del turista y se basa, fundamentalmente, en los intereses de marketing de estos proveedores de información. Ello se explica por la importancia que adquiere en la toma de decisiones durante la fase pre-vacacional la percepción del clima/tiempo del destino, frente al menor peso que adquiere la realidad atmosférica del lugar.

En cuanto al cumplimiento del criterio de utilidad señalado por la OMM, el análisis cualitativo aplicado en este trabajo ha evidenciado que la mayor parte de la información climático-meteorológica proporcionada por los organismos encargados de la promoción de los destinos turísticos españoles no se encuentra adaptada a las necesidades del turista. En este sentido, cabe señalar que la información es muy generalista y escueta, no siempre cubre satisfactoriamente todos los estadios de la experiencia turística y pocas veces se encuentra adecuada a la modalidad susceptible de ser practicada por el visitante (deportes náuticos, sol y playa, esquí, senderismo, etc.) o al tipo de entorno en el que se lleva a cabo la actividad (costa, montaña, urbano, etc.). Tampoco se muestra información integrada, ni valores probabilísticos, ni se ofrecen consejos que permitan aprovechar los beneficios del clima/tiempo o atenuar algunos efectos nocivos derivados del mismo (tampoco se proporcionan alertas meteorológicas). Los proveedores públicos y privados de información meteoclimática también presentan debilidades en cuento a la utilidad de la información pero en menor grado que los organismos promotores. Estos portales ofrecen información meteorológica de interés para el visitante turístico, pero la misma no siempre se encuentra sistematizada, ni adaptada a sus necesidades, al menos de forma gratuita. En cuanto a esto último, cabe señalar que si bien en todos estos portales quedan cubiertas las necesidades de información para diferentes momentos horarios y días vista, rara vez se incorporan pronósticos meteorológicos basados en índices de aptitud climático-turística, que permitan la valoración de una jornada para distintas actividades y subsectores turísticos a través de una valoración numérica de 0 a 10 .

La información climático-meteorológica suministrada por los organismos encargados de la promoción de los destinos turísticos españoles adquiere frecuentemente el formato descriptivo-verbal. En éste abundan la generalización y la imprecisión, lo que resta veracidad y, de nuevo, utilidad a la información transmitida. No sucede lo mismo con los organismos públicos y privados que proporcionan estos servicios: se proporcionan cuantiosos valores numéricos, plasmación icónica de la 
información y descripciones, en ocasiones, excesivamente técnicas que dificultan un rápido entendimiento por parte del turista.

La temperatura y la insolación son los atributos dominantes en la información climático-meteorológica facilitada por los organismos encargados de la promoción de los destinos. Sin embargo, parámetros que pueden ser de interés para el turista (sensación térmica, índice UV, temperatura del agua, oleaje, etc.) no son contemplados en esa transmisión, al menos de forma generalizada. En este sentido, cabe señalar que la información transmitida puede mejorar la toma de decisiones en aspectos relacionados con la satisfacción de la necesidad de disfrute que presenta el turista, pero rara vez con la necesidad de confort o seguridad. No sucede lo mismo en los organismos dedicados a la prestación de servicio meteo-climáticos: el número de parámetros abordados y el detalle bruto de la información (no se encuentra integrada en tipos de tiempo) puede superar en ocasiones los requerimientos de un turista medio.

Si bien las TICs se han incorporado con fuerza al sector turístico y al sector de los servicios ambientales, desempeñando un importante papel en la promoción de los destinos y en la transmisión de información, se observan ciertas debilidades, al menos en lo relativo al tema que aquí nos ocupa. En este sentido, cabe señalar que a pesar del crecimiento en el número de aplicaciones vinculadas al turismo y al tiempo, todavía son pocas las centradas total o parcialmente en aspectos climático-turísticos y que, además, sean accesibles desde los portales promocionales o los proveedores de servicios meteo-climáticos. Este tipo de Apps podrían ser de gran utilidad en la preparación del viaje y en la interacción e integración del turista en el destino, por lo que se podrían constituir en herramientas fundamentales para la mejora de la experiencia del visitante. Por el contrario, las herramientas de la Web 2.0 si se encuentran mayoritariamente incorporadas en los portales, hecho que facilita la transmisión de información por parte de los propios protagonistas de la experiencia turística. Una información que ejerce una gran influencia en la toma de decisiones de otros turistas, ya que aportan objetividad en la descripción de los destinos en comparación con la información promocional.

En el contexto del turismo, proporcionar información climático-meteorológica significa difundir datos procesados, veraces y adaptados a los diferentes usuarios potenciales. En este sentido cabe indicar que si bien existe una amplia gama de información ambiental disponible, su utilidad se ve seriamente reducida, dadas las formas en las que se presenta (De Freitas, 2003; Grifoni, 2004; Scott y Lemieux, 2010). Por ello, el abasto de información meteo-climática existente no suele ser bien aprovechada por los operadores turísticos (Wall, 2003). El turista requiere información diferenciada en las distintas fases de la experiencia turística, ajustada a sus necesidades y con la debida resolución temporal y espacial.

Mejorar el nivel de la información atmosférica con fines turísticos se configura como un gran reto que solicitará la colaboración entre los investigadores del binomio clima-turismo, los servicios meteorológicos estatales y autonómicos, las autoridades de turismo y la propia industria turística. Mejorar la difusión de la información atmosférica con fines turísticos también se configura como un gran desafío que requerirá la constante incorporación de nuevas TICs. Ello facilitará la preparación del 
viaje, la interacción e integración del turista en el destino y la mejora de la experiencia turística, aspectos clave de los llamados nuevos destinos turísticos inteligentes.

\section{BIBLIOGRAFÍA}

ALDEANUEVA FERNÁNDEZ, I. y JIMÉNEZ QUINTERO, J.A. (2012). Las tecnologías de la información y la comunicación en las pymes turísticas andaluzas: Una perspectiva estratégica de clúster. Cuadernos de CC.EE. y EE., 62, 37-48.

ALTALO, M. y HALE, M. (2002). Requirements of the US Recreation and Tourism Industry for Climate, Weather and Ocean Information. Consultants report to NOAA.

ANDREU, L., BIGNE, J.E. y COOPER, C. (2000). Projected and perceived image of Spain as a tourist destination for British travelers. Journal of Travel \& Tourism Marketing, 9(4), 47-67.

ANTÓN CLAVÉ, S. y VILLALTA, E. (2004). La presencia en Internet de los principales destinos turísticos del litoral Mediterráneo español, en TURITEC 2004, V Congreso Nacional de Turismo y Tecnologías de la Información y las Comunicaciones. Málaga, Pub. Universidad de Málaga, 1-18.

BECKEN, S. (2010). The importance of climate and weather for tourism: literature review. Lincoln University, LEaP.

BECKEN, S. y HAY, J. (2007). Tourism and Climate Change - risks and opportunities. Clevedon, Channel View Publications.

BECKEN, S. y WILSON, J. (2013). The impacts of weather on tourist travel. Tourism Geographies, 15(4), 620-639.

BIGANO, A., GORIA, A., HAMILTON, J.M. y TOL, R.S.J. (2005). The Effect of Climate Change and Extreme Weather Events on Tourism (February 1, 2005). Working Paper No. 30.05; CMCC Research Paper No. 01. FEEM. Available at SSRN: http://ssrn.com/abstract=673453 or http://dx.doi.org/10.2139/ssrn.673453

BIGANO, A., HAMILTON, J.M. y TOL, R.S.J. (2006). The impact of climate on holiday destination choice. Climatic Change, 76, 389-406.

CHUNG, J.Y. y BUHALIS, D. (2008). Web 2.0: A study of online travel community. En ENTER 2008, 70-81.

DE FREITAS, C.R. (2003). Tourism climatology: evaluating environmental information for decision making and business planning in the recreation and tourism sector. International Journal of Biometeorology, 48, 45-54.

EUGENIO-MARTIN, J.L. y CAMPOS-SORIA, J.A. (2010). Climate in the region of origin and destination choice in outbound tourism demand. Tourism Management, 31, 744-753.

GÓMEZ MARTíN M.B. (1999). El Clima como activo del turismo: los folletos turísticos catalanes, en El Territorio y su Imagen (vol. 1). Málaga, Universidad de Málaga y Consejería de Medio Ambiente de la Junta de Andalucía.

GÓMEZ MARTÍN, M.B. (2005). Weather, Climate and Tourism. A Geographical Perspective. Annals of Tourism Research, 32(3), 571-591. 
GÓMEZ MARTÍN, M.B. y MARTÍNEZ IBARRA, E. (2012). Tourism demand and atmospheric parameters: Non-intrusive observation techniques. Climate Research, Vol. 51, 135-145.

GÓMEZ MARTÍN, M.B., ARMESTO LÓPEZ, X. y MARTÍNEZ IBARRA, E. (2013). The Spanish tourist sector facing extreme climate events: a case study of domestic tourism in the heat wave of 2003. International Journal of Biometeorology, (aceptado, online first DOI 10.1007/s00484-013-0659-6).

GÖSSLING, S., SCOTT, D., HALL, C.M., CERON, J.P. y DUBOIS, G. (2012). Consumer Behaviour And Demand Response Of Tourists To Climate Change. Annals of Tourism Research, 39(1), 36-58.

GRIFONI, D. (2004). Developing an operational system to support tourism activities in tuscany region. En Matzarakis, A., De Freitas, C.R. y Scott, D. (Eds.): Advances in Tourism Climatology. Freiburg, 166-173.

HALL, C. M. y HIGHAM, J. (2005). Tourism, recreation and climate change. Clevedon, Channel View Publications.

HAMILTON, J.M. y LAU, M. (2005). The Role of Climate Information in Tourist Destination Choice Decision-Making. En Gössling, S. y Hall, C.M. (Eds.): Tourism and Global Environmental Change. London: Routledge, 229-250.

HU, Y. y RITCHIE, J. (1993). Measuring destination attractiveness: A contextual approach. Journal of Travel Research, 32, 25-34.

HUERTAS, A. (2008). Aplicación de la Web 2.0 a los destinos turísticos. Implantación y diferencias. En TURITEC 2008, VII Congreso Turismo y Tecnologías de la Información y las Comunicaciones. Málaga, Pub. Universidad de Málaga, 1-16.

IVARS, J.A. (2013). Destinos Turísticos Inteligentes. En AECIT 2012, La actividad turística española en 2012 (Edición 2013). Madrid, Editorial Centro de Estudios Ramón Areces, 473-476.

LIM, G.G., KIM, D.H., CHOI, M., CHOI, J.H. y LEE, K.C. (2010). An exploratory study of the weather and calendar effects on tourism web site usage. Online Information Review, 34(1), 127-144.

LOOKINSIDE.TRAVEL (2013). Estudio sobre el viajero español 2012. http://www.lookinside.travel/resources/presentacion-evento-2012.pdf

MARTÍNEZ IBARRA, E. y GÓMEZ MARTÍN, M.B. (2012). Weather, climate and Tourist behaviour. The beach tourism of the Spanish Mediterranean coast as a case study. European Journal of Tourism, Hospitality and Recreation, 3, 77-96.

MARTÍNEZ IBARRA, E. y GÓMEZ MARTÍN, M.B. (2012). Progress in Tourism Climatology. En Mihalic, T. and Gartner, W.C. (Ed.): Tourism and Developments. Issues and Challenges. Nova Science Publishers, 121-138.

MCKERCHER, B., SHOVAL, N., PARK, E. y KAHANI, A. (2014). The [Limited] Impact of Weather on Tourist Behavior in an Urban Destination. Journal of Travel Research (aceptado, online first DOI 0047287514522880).

MORENO, A., AMELUNG, B. y SANTAMARTA, L. (2008). Linking beach recreation to weather conditions: A case study in Zandvoort, Netherlands. Tourism in Marine Environments, 5(2-3), 111-119. 
NIELSEN (2011). Nielsen Mobile Insigh Spain. Nielsen España.

OMM (2008). Boletín, 57(4). Organización Meteorológica Mundial.

OMM (2012). Boletín, 61(2). Organización Meteorológica Mundial.

PIKE, S. (2002): Destination image analysis - A review of 142 papers from 1973 to 2000. Tourism Management, 23, 541-549.

RICO-AMOROS, A.M., OLCINA-CANTOS, J. y SAURÍ, D. (2009). Tourist land use patterns and water demand: evidence from the western Mediterranean. Land Use Policy, 26, 493-501.

RIDDERSTAAT, J.; ODUBER, M.; CROES, R.; NIJKAMP, P. y MARTENS, P. (2014). Impacts of seasonal patterns of climate on recurrent fluctuations in tourism demand: Evidence from Aruba. Tourism Management, 41, 245-256.

SCOTT, D., WALL, G. y MCBOYLE, G. (2005). The evolution of the climate change issue in the tourism sector. In Hall, C. M. y Higham, J. (Eds.): Tourism, recreation and climate change. Clevedon, Channel View Publications.

SCOTT, D., AMELUNG, B., BECKEN, S., CERON, J.-P., DUBOIS, G., GOSSLING, S., PEETERS P. y SIMPSON, M. (2008). Climate Change and Tourism: Responding to Global Challenges. United Nations World Tourism Organization.

SCOTT, D. y LEMIEUX, C. (2010). Weather and Climate Information for Tourism. Procedia Environmental Sciences, 1, 146-183.

SHIH, C., NICHOLLS, S. y HOLECEK, D. (2009). Impact of weather on downhill ski lift ticket sales. Journal of Travel Research, 47, 359-372.

WALL, G. (2003). The tourism industry and its adaptability and vulnerability to climate change. En Climate Change and Tourism: Assessment and Coping Strategies, Warsaw.

WORLD METEOROLOGICAL ORGANIZATION (WMO) (2002). WMO questionnaire on the role and operation of National Meteorological Services: Some findings. WMO Bulletin 51:342. 